\title{
Post-chikungunya arthritis: a longitudinal study in a tertiary care hospital in Bangladesh
}

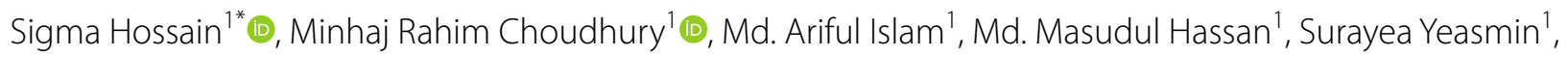
Farzana Hossain ${ }^{2}$ and Mohammad Mostafa Zaman ${ }^{3}$ (D)

\begin{abstract}
Background and objective: To identify the clinical patterns and consequences of post-chikungunya arthritis was the study's objective.

Methods: This longitudinal study was carried out among 143 Chikungunya virus (CHIKV) infected adult patients at the rheumatology department, Bangabandhu Sheikh Mujib Medical University (BSMMU), Dhaka, Bangladesh, during the outbreak of CHIKV infection in 2017. The disease was categorized into three phases: acute or febrile (lasting up to 10 days), subacute (11-90 days), and chronic (>90 days). Patients who progressed towards the chronic phase were followed up to 1-year. Post-CHIKV de novo chronic inflammatory rheumatisms (CIRs) were characterized by persistent mono or oligoarthritis, undifferentiated polyarthritis, or meet the criteria rheumatoid arthritis (RA) or Spondyloarthritis (SpA). In addition, functional status was assessed by the validated Bangla version of the Health Assessment Questionnaire (HAQ).
\end{abstract}

Results: Mean age was $43.3 \pm 11.5$ years, and $51.0 \%$ were male. Within 1-year follow-up, 60 (41.9\%) patients were suffering from arthralgia/ arthritis. Of them 52 patients did not have any pre-existing arthralgia/arthritis. 35 (65.3\%) had undifferentiated arthritis, 10 (19.2\%) had SpA, and 7 (13.5\%) had RA. Patients with pre-existing rheumatological disorders, 6(4.2\%) had SpA, $1(0.7 \%)$ had RA and $1(0.7 \%)$ had osteoarthritis. Polyarthralgia $(n=33,55.0 \%)$ and polyarthritis $(n=20,33.3 \%)$ were the main presentations. Female gender (OR: $0.45 ; \mathrm{Cl}: 0.21-0.96)$, positive IgG (OR: 0.30; $\mathrm{Cl}: 0.12-0.76)$, and moderate to severe functional disability (OR: 3.46; $\mathrm{Cl}: 1.62-7.40)$ were independent predictors of developing chronic post-CHIKV rheumatism.

Conclusions: At 1-year follow-up, more than one-third of the patients remained symptomatic. Female gender, positive $\mathrm{lgG}$, and moderate to severe functional disability contributed to the development of chronicity.

Keywords: Chikungunya infection, Post-chikungunya arthritis, Health assessment questionnaire (HAQ), Longitudinal study, Bangladesh

\section{Significance \& innovations}

- This is the first longitudinal study conducted on Chikungunya (CHIK) arthritis patients in a tertiary care hospital during the significant outbreak of CHIK in

*Correspondence: sigma_hossain@hms.harvard.com

${ }^{1}$ Department of Rheumatology, Bangabandhu Sheikh Mujib Medical University (BSMMU), Shahbag Avenue, Dhaka, Bangladesh

Full list of author information is available at the end of the article
Bangladesh, and all patients were evaluated and diagnosed by rheumatologists.

- While most patients recover from acute chikungunya virus infection within days or weeks, some patients develop chronic and incapacitating joint morbidities due to this virus infection.

- Risk factors for developing chronic post-CHIKV rheumatism includes female gender, positive IgG, and moderate to severe functional disability contributed to the development of chronicity. original author(s) and the source, provide a link to the Creative Commons licence, and indicate if changes were made. The images or other third party material in this article are included in the article's Creative Commons licence, unless indicated otherwise in a credit line to the material. If material is not included in the article's Creative Commons licence and your intended use is not permitted by statutory regulation or exceeds the permitted use, you will need to obtain permission directly from the copyright holder. To view a copy of this licence, visit http://creativecommons.org/licenses/by/4.0/. 


\section{Introduction}

Chikungunya (CHIK) is a mosquito borne (Aedes species) febrile illness caused by the Chikungunya virus (CHIKV). It was first identified during an epidemic of febrile polyarthralgia in Tanzania in 1953 [1]. Since then, outbreaks have been attributed to many Southeast Asia and Indian countries. The disease became known worldwide after a major epidemic occurred in 2005 on Reunion Island, France, when more than $30 \%$ of the population of this island was affected by Chikungunya fever (CHIKF) [2].

In 2008, the Institute of Epidemiology, Disease Control and Research (IEDCR), Bangladesh, and the International Centre for Diarrhoeal Disease Research, Bangladesh (ICDDR, B) identified and investigated the first outbreak Chikungunya fever in Rajshahi and Chapianawabganj districts of Bangladesh [3]. Two small-scale outbreaks were documented in rural communities in 2011 [4] and 2012 [5]. Dhaka, one of the most densely populated cities globally with approximately 18 million inhabitants [6], has experienced the Chikungunya outbreak in 2017.

Chikungunya virus (CHIKV) infection has an incubation period of 2-7 days [7]. CHIKF is characterized by an acute phase followed by subacute and chronic phases. Acute CHIKF is often accompanied by high fever, headache, maculopapular rash, myalgias, and severe arthritis/arthralgias. Exanthema and polyarthralgia primarily affect the hands and feet, which cause significant functional disabilities [8-10].

The subacute phase lasts between 11 days and 3 months. After 3 months, the chronic form can persist for 6 years [11]. Systematic reviews and meta-analyses had shown that approximately $25 \%$ of Chikungunya cases would develop chronic inflammatory rheumatism, and $14 \%$ would develop chronic arthritis [12]. McCarthy and Morrison cited age above 45, female gender, and the existence of prior musculoskeletal diseases as clinicalepidemiological risk factors for the chronicity of musculoskeletal complications in CHIKF [13].A South Indian study also represented similar risk factors [14]. In a small percentage of patients, a condition that resembles rheumatoid arthritis (RA) may develop, possibly caused by the presence of the HLA-DRB1 gene (associated with the development of RA) in these patients. However, unlike classic RA, high levels of rheumatoid factor and anticyclic citrullinated peptides are not detected [15]. The most common ultrasonographic findings were synovitis, tenosynovitis [16].

Several cross-sectional studies and case series have been reported in Bangladesh [17, 18]. Hossain et al. found joint pain and fever were common among the patients. Arthralgia was polyarticular type in $56.3 \%$ of the patients. About $83 \%$ of the patients reported low to very low overall quality of life. Nearly $30 \%$ of the patients lost more than 10 days of productivity due to severe arthropathy [19]. Unfortunately, no longitudinal study was done regarding Chikungunya infection, focusing on the enduring effect of the disease among the Bangladeshi population. Therefore, the present study had been undertaken to identify the clinical patterns and consequences of post-chikungunya arthritis.

\section{Materials and methods \\ Study design}

This longitudinal study was carried out among 143 CHIKV infected patients (IgM and/or IgG positive) at the outpatient department of Rheumatology, BSMMU, Dhaka, Bangladesh, during the outbreak of CHIKV infection in 2017. The study followed the Strengthening the Reporting of Observational Studies in Epidemiology (STROBE) guideline [20].

\section{Subject enrollment}

Patients who experienced typical clinical symptoms of CHIKV infection (febrile illness with arthralgia/arthritis) during the peak of the recent Dhaka outbreak (May-September 2017) were enrolled in this study. For confirmed case, we followed the recommendations of the World Health Organization (WHO) [21] where during an established outbreak, a patient fulfilling laboratory criteria (detection of positive RT-PCR or IgM antibody and/or demonstration of rising titre of IgG antibody for CHIKV but not dengue) irrespective of the clinical presentation were considered as a confirmed case. On the other hand, patients who claimed to have CHIKV infection without physicians' confirmation (clinical and or laboratory) were excluded from the study. As a result, we had only confirmed cases. The disease was categorized into three phases: acute or febrile (lasting up to 10 days), subacute (11-90 days), and chronic (>90 days) [22].

Post-Chikungunya- Chronic Inflammatory Rheumatism (pCHIK-CIR) was characterized by persistent mono or oligoarthritis, undifferentiated polyarthritis, or meet criteria for Rheumatoid Arthritis or Spondyloarthritis [23].

Patient who fulfills the American College of Rheumatology (ACR)/European League Against Rheumatism (EULAR) Classification Criteria 2010 criteria [24] was considered having rheumatoid arthritis.

A patient who fulfills Spondyloarthritis (axial and peripheral): Ankylosing Spondylitis Assessment Study (ASAS) criteria 2011 [25] and/or Ankylosing spondylitis: Modified New York Criteria 1984 was considered having Spondyloarthritis [26].

The term "undifferentiated arthritis" (UA) is used here to describe patients with early inflammatory arthritis, 
typically between six weeks and a year in duration, whose disease cannot yet be diagnosed or differentiated from other defined disorders. However, a diagnosis can often be determined within three months and only infrequently requires a year to become evident. Many such patients will eventually be diagnosed with rheumatoid arthritis (RA) after further evolution of the symptoms and findings [27].

\section{Patient assessment}

Meticulous history taking and thorough physical examination were done on every patient after enrollment in the study. For each, 68 joints were examined. In addition, clinical, routine laboratory (complete blood count, CRP, etc.) and musculoskeletal ultrasonographic features were evaluated in BSMMU by a group of rheumatologists trained in musculoskeletal ultrasonography using the grayscale with a linear $15 \mathrm{MHz}$ multifrequency transducer in B-mode and with power doppler (PD) $(750 \mathrm{~Hz}$ PRF, low wall filter and gain adjusted just below the appearance of artifacts) (Machine: High-End Radiology Ultrasound System, Mode: Logiq 5 Pro [GE Healthcare]). Ultrasonography scanning technique, grayscale, PD machine setting, and definitions of abnormalities were standardized according to EULAR recommendations for reporting ultrasound studies in rheumatic and musculoskeletal diseases among investigators before the study [28].
Synovitis, tenosynovitis, and bursitis were defined according to Naredo et al. Synovial, tenosynovial, and intrabursal blood flow at each joint was evaluated PDUS. PD imaging was performed by selecting a region of interest that included the bony margins, synovial site, and a variable view of surrounding tissues [29]. When thickened median nerves found in ultrasound were accompanied by hand paresthesia, it was suggestive of carpal tunnel syndrome (CTS) [16].

Functional status was assessed by the validated Bengali version of the Health Assessment Questionnaire (HAQ) [30]. The HAQ-DI is scored from 0.0 (no functional disability) to 3.0 (marked functional disability), where 0.0 to 1.0 is none to mild functional disability, 1.1 to 2.0 is a moderate functional disability, and 2.1 to 3.0 is severe functional disability [31].

Patients who have post-CHIK arthritis were followed up after one month and 3 months from the date of enrollment for documenting their clinical improvement. In addition, rheumatoid factor, ACPA, HLAB27, X-ray sacroiliac joint were performed according to the requirement of patient condition. Patients who progressed towards the chronic phase (more than three months) were followed up to one year (Fig. 1).

\section{Data analysis}

Data were collected through a face-to-face interview in a pre-designed semi-structured questionnaire. Data were analyzed by using the SPSS-26 version. Descriptive

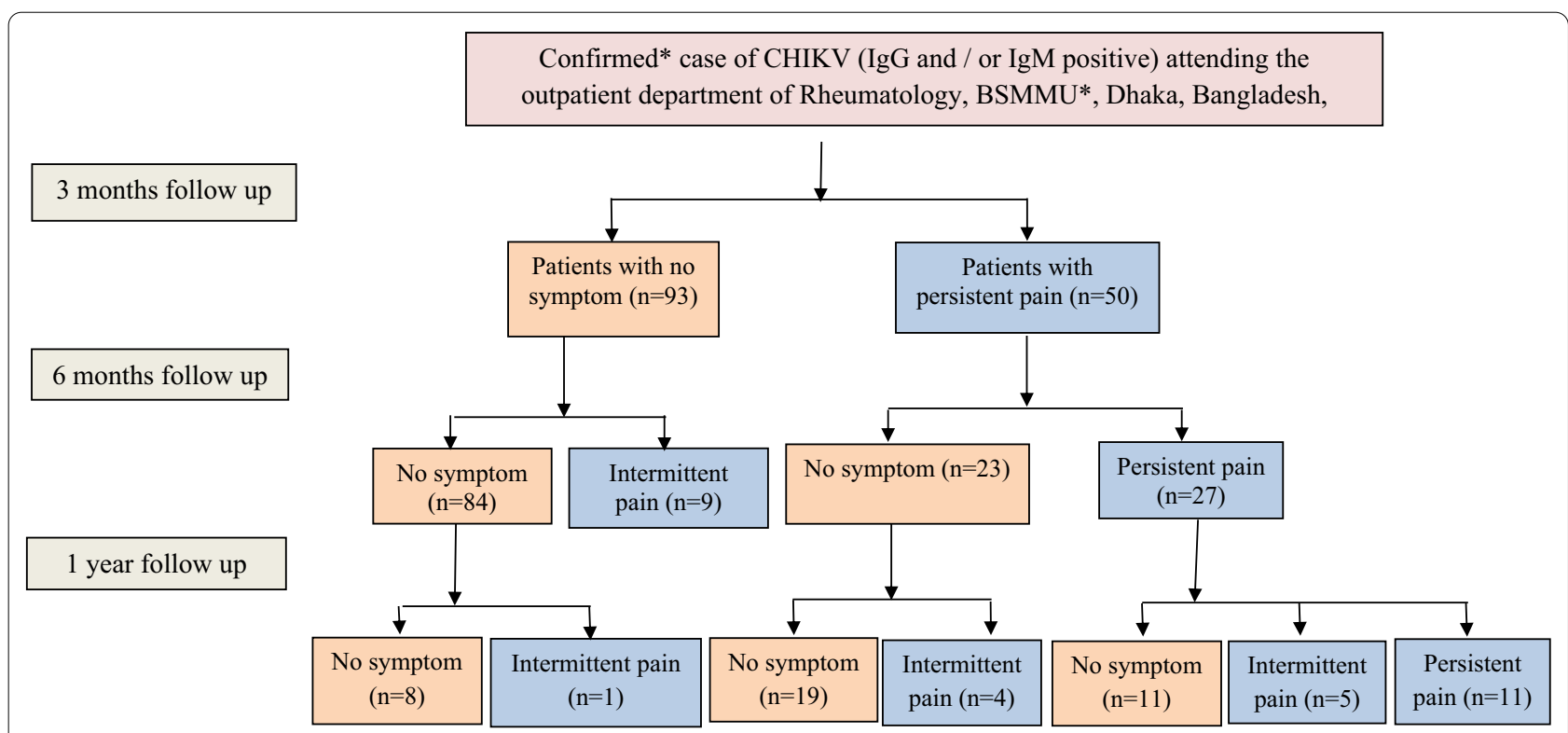

Fig. 1 Study participants have been grouped into one image. ${ }^{*} B S M M U$ Bangabandhu Sheikh Mujib Medical University. ${ }^{*}$ The patient fulfilling the laboratory criteria \{detection of positive reverse transcription-polymerase chain reaction or lgM antibody and/or demonstration of a rising titer of IgG antibody for Chikungunya virus (CHIKV) but not dengue\}, regardless of the clinical presentation, is considered a confirmed case 
statistical analysis included mean and standard deviation (SD) for continuous data and frequency and proportions for categorical data. For skewed data, a median with an interquartile range was used. While finding predictors of pCHIK-CIR, patients with intermittent or persistent pain after 3 months of acute pain were considered to have pCHIK-CIR. Associations of categorical data were assessed using the Chi-squared test, where $P$ values less than 0.05 were considered significant. Crude odds ratios (OR) with 95\% confidence interval (CI) were calculated. Gender, comorbidity, IgG, and functional disability were selected for the logistic model. Stepwise Logistic Regression was performed using backward elimination. Adjusted OR and 95\% CI were calculated as part of the logistic procedure. It was mentionable that patients with pre-existing rheumatologic disorders were included in the logistic regression analysis as pre-existing rheumatologic diseases were found not to influence the pattern of joint involvement, type of arthritis, and pain characteristics on chronic patients.

\section{Ethical consideration}

Study approval was obtained from the Institutional Review Board of BSMMU (BSMMU/2017/11262). Informed written consent was taken from the adult patients. A complete assurance was given that all information would be kept confidential. The right was being given to the patients not to participate and to discontinue participation at any time in the study with consideration/ without penalty. The Declaration of Helsinki's ethical guidelines were followed in the study.

\section{Results}

The mean age of the patients was $43.3 \pm 11.5$ years, where $44(30.8 \%)$ were from 31 to 40 , and $43(30.1 \%)$ were from the $41-50$ years group. Seventy-three $(51.0 \%)$ patients were male, 55 (38.5\%) patients were service holders, and $53(37.1 \%)$ were housewives. One hundred and thirty-six (95.1\%) patients were urban residents. Comorbidity was present in $59(41.3 \%)$ patients were $34(23.8 \%)$ patients were hypertensive, and 30 (20.9\%) were diabetic. The preexisting rheumatologic disorder was present among 8 (5.6\%) patients (Table1).

Clinical features of the patients showed that all patients had a fever, $121(84.6 \%)$ had symmetrical joint involvement, wherein 96 (67.1\%) patients the involvement was generalized at the onset. Subsequently, ankle joint was mainly affected in 119 (83.2\%) patients. Metacarpophalangeal (MCP) joint, wrist, Proximal interphalangeal (PIP) joints, and knee joints were affected in 100 (69.9\%), 97 (67.8\%), 84 (58.7\%), and $76(53.1 \%)$ patients, respectively. In $68(47.6 \%)$ and $117(81.8 \%)$ patients, joint swelling and morning stiffness were
Table 1 Socio-demographic features of the patients $(n=143)$

\begin{tabular}{lc}
\hline Socio-demographic features & $\boldsymbol{n}(\%)$ \\
\hline Age (in years) & \\
$20-29$ & $18(12.6)$ \\
$30-39$ & $36(25.2)$ \\
$40-49$ & $43(30.1)$ \\
$50-71$ & $46(32.2)$ \\
Gender & \\
Men & $73(51.0)$ \\
Women & $70(49.0)$ \\
Educational status & \\
No formal education (0) & $15(10.5)$ \\
Any primary education (1-5) & $37(25.9)$ \\
Any secondary education (6-10) & $20(14.0)$ \\
Higher secondary (11-12) & $20(14.0)$ \\
Above higher secondary (>12) & $51(35.7)$ \\
Occupational status & \\
Service holder & $55(38.5)$ \\
Homemaker & $53(37.1)$ \\
Independent (businessman and day labourer) & $26(40.6)$ \\
Others (student and retired person) & $9(6.3)$ \\
Residential area & \\
Urban & $136(95.1)$ \\
Rural & $7(4.9)$ \\
Co-morbidity & $59(41.3)$ \\
Hypertension & $34(23.8)$ \\
Diabetes mellitus & $30(20.9)$ \\
Bronchial asthma & $4(2.8)$ \\
Hypothyroidism & $4(2.8)$ \\
Pre-existing spondyloarthritis & $1(0.7)$ \\
Pre-existing osteo-arthritis & $1(0.7)$ \\
\hline
\end{tabular}

present, respectively. Myalgia was present in 47 (32.9\%) patients, and skin involvement was present in 91 (63.6\%) patients, where 67 (46.9\%) patients had pruritic, erythematous rash. Forty-nine (34.3\%) patients had moderate, while $15(10.5 \%)$ patients had a severe functional disability. Laboratory findings showed that IgM was present in 135 (94.4\%) patients, and IgG was present in $29(20.3 \%)$ patients. Out of 143 patients, an investigation report for $\mathrm{C}$-reactive protein (CRP) was present for 48 patients where $39(81.2 \%)$ had high CRP (>6 mg/l). Median ESR was $31.0[15.0,45.0] \mathrm{mm} /$ hour (Table 2).

The total disability index of the patients was 1.4 [0.7, 1.9] where dressing had the lowest scores $1.0[0.0,1.0]$. Walking, hygiene, and common daily activities had 
Table 2 Clinical features and laboratory findings of the patients in acute stage $(n=143)$

\begin{tabular}{|c|c|}
\hline Clinical features & $n(\%)$ \\
\hline Fever & $143(100)$ \\
\hline Symmetrical joint involvement & $121(84.6)$ \\
\hline \multicolumn{2}{|l|}{ Joint involvement at onset } \\
\hline Generalized & $96(67.1)$ \\
\hline Ankle & $22(15.4)$ \\
\hline Knee & $15(10.5)$ \\
\hline Others & $10(6.9)$ \\
\hline \multicolumn{2}{|l|}{ Subsequent joint involvement } \\
\hline Ankle & $119(83.2)$ \\
\hline Metacarpophalangeal joint & $100(69.9)$ \\
\hline Wrist & $97(67.8)$ \\
\hline Proximal interphalangeal joints & $84(58.7)$ \\
\hline Knee & $76(53.1)$ \\
\hline Shoulder & $64(44.8)$ \\
\hline Elbow & $51(35.7)$ \\
\hline Metatarsophalangeal joint & $46(32.2)$ \\
\hline Distal interphalangeal joints & $43(30.1)$ \\
\hline Others (hip, temporomandibular joint, axial plane) & $30(20.9)$ \\
\hline Joint swelling & $68(47.6)$ \\
\hline Morning stiffness & $117(81.8)$ \\
\hline Myalgia & $47(32.9)$ \\
\hline Skin involvement & $91(63.6)$ \\
\hline \multicolumn{2}{|l|}{ Pattern of skin involvement } \\
\hline Pruritic erythematous rash & $67(46.9)$ \\
\hline Erythematous rash & $24(16.8)$ \\
\hline \multicolumn{2}{|l|}{ Functional disability } \\
\hline Mild & $79(55.2)$ \\
\hline Moderate & $49(34.3)$ \\
\hline Severe & $15(10.5)$ \\
\hline \multicolumn{2}{|l|}{ Laboratory findings } \\
\hline Positive Immunoglobulin M (IgM) & $135(94.4)$ \\
\hline Positive Immunoglobulin G (lgG) & $29(20.3)$ \\
\hline C-reactive protein (CRP) positive $(n=48)$ & $39(81.2)$ \\
\hline $\begin{array}{l}\text { Erythrocyte sedimentation rate ( } \mathrm{mm} \text { in 1st hour), } \\
\text { median [IQR] }\end{array}$ & $31.0[15.0-45.0]$ \\
\hline White blood cells count $\left(10^{3} / \mu \mathrm{L}\right)$, mean $\pm \mathrm{SD}$ & $8.4 \pm 2.4$ \\
\hline Platelet count $\left(10^{3} / \mu \mathrm{L}\right)$ mean $\pm \mathrm{SD}$ & $297.1 \pm 91.7$ \\
\hline Serum creatinine (mg/dl) & $0.8 \pm 0.2$ \\
\hline
\end{tabular}

*IQR Inter-quartile range

highest scores $2.0[1.0,2.0], 2.0[1.0,3.0]$ and $2.0[1.0$, 3.0 ] respectively (Table 3 ).

Joint swelling $(p=0.004)$, skin involvement $(p<0.001)$, and functional disability $(p=0.015)$ were significantly more common in females (Fig. 2).

60 (41.9\%) patients developed post-ChikungunyaChronic inflammatory rheumatism (pCHIK-CIR) within the one-year follow-up. Pain without swelling was
Table 3 The Health Assessment Questionnaire Disability Index (HAQ-DI) Scores of the patients in acute stage $(n=143)$, median [Q1,Q3]

\begin{tabular}{ll}
\hline HAQ-DI Scores & Median [Q1,Q3] \\
\hline Dressing & $1.0[0.0,1.0]$ \\
Arising & $1.0[0.0,2.0]$ \\
Eating & $1.0[0.0,2.0]$ \\
Walking & $2.0[1.0,2.0]$ \\
Hygiene & $2.0[1.0,3.0]$ \\
Reach & $1.0[1.0,2.0]$ \\
Grip & $1.0[0.0,2.0]$ \\
Common daily activities & $2.0[1.0,3.0]$ \\
Total score & $1.4[0.7,1.9]$ \\
\hline
\end{tabular}
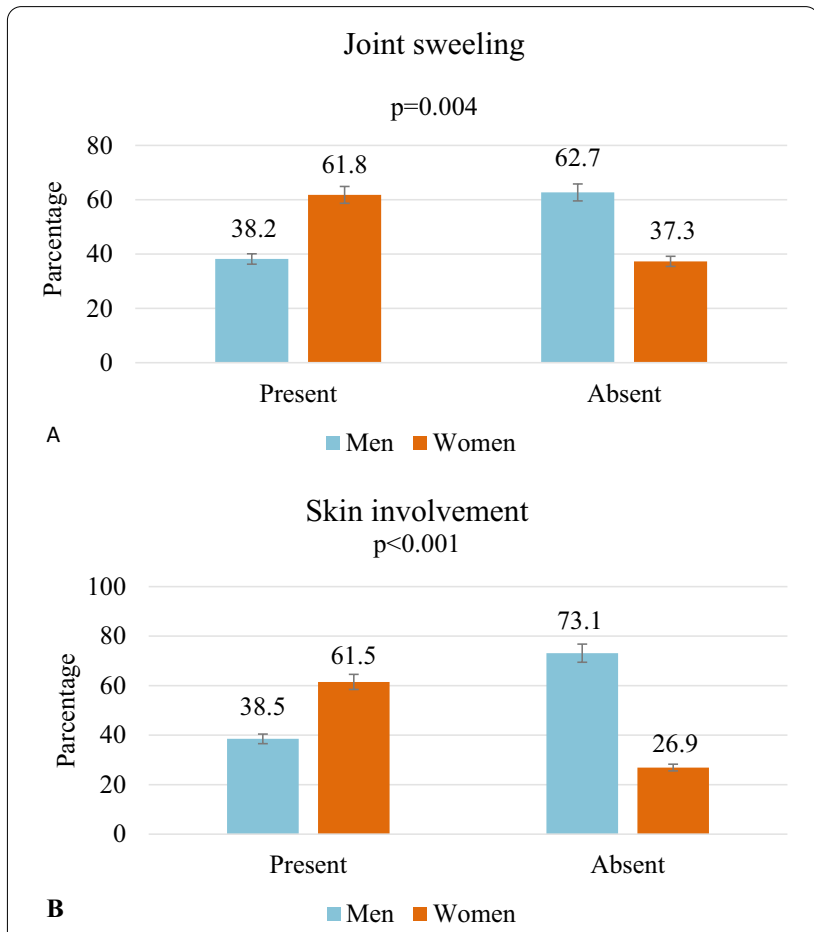

Functional disability $\mathrm{p}=0.015$

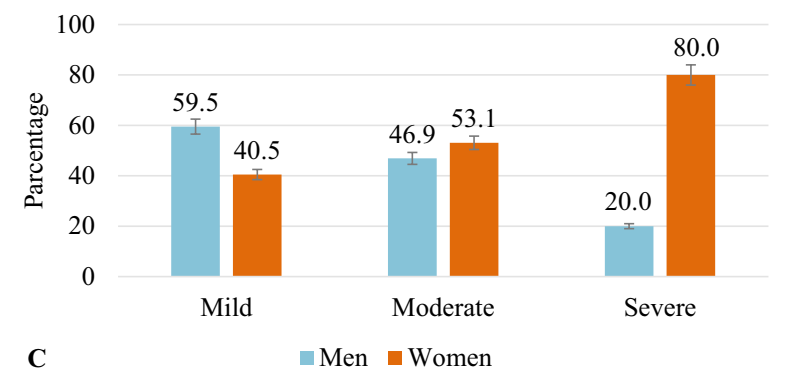

Fig. 2 Joint swelling (A), skin involvement (B) and functional disability (C) of patients according to gender $(n=143)$ 
present in 39 (65.0\%) patients. Ultra-sonographic findings showed that tenosynovitis was present in 19 (31.7\%) patients, while median nerve entrapment was present in 4 (6.7\%) patients. At the end of one year, HAQ score was available for 21 patients where 18 (85.7\%) patients had a mild functional disability. No significant statistical difference was observed between patients with or without the pre-existing rheumatologic disorder (except tenosynovitis) and between males and females regarding characteristics of chronic patients (Table 4).

Among the 60 CHIK patients, 35 had undifferentiated arthritis, where polyarthralgia was present in 21 (60.0\%) patients, and polyarthritis was present in $8(22.8 \%)$ patients. Ten patients had spondyloarthritis where polyarthralgia was present in $6(60.0 \%)$ patients, and seven patients had rheumatoid arthritis where 5 (71.4\%) had polyarthritis (Table 5).
At 3 months, in $23(46.0 \%)$ patients, polyarthralgia and oligoarthritis were found in $10(20.0 \%)$ patients. At 6 months, polyarthralgia was noted in 18 (50.0\%) patients, and polyarthritis was reported in 9 (11.1\%) patients. At one year, polyarthralgia was found in 11 (52.4\%) patients, and only one patient (4.8\%) had polyarthritis (Fig. 3).

Patients with comorbidity had significantly more chances of having pCHIK-CIR (Crude OR: 2.10; CI: $1.06-4.15, p=0.039)$. Patients having positive IgG (Crude OR: 3.38; CI: $1.44-7.96,=0.006$ ) and moderate to severe functional disability (Crude OR: 0.34; CI: 0.17-0.69) also had significantly more chance of having pCHIK-CIR. Stepwise logistic regression using backward elimination showed that female gender (adjusted OR: 0.45; CI: $0.21-0.96, p=0.039$ ), positive Ig G (adjusted OR: 0.30; CI: 0.12-0.76, $p=0.011$ ) and

Table 4 Characteristics of the patients with Chronic Chikungunya arthritis $(n=60)$

\begin{tabular}{|c|c|c|c|c|c|}
\hline \multirow[t]{2}{*}{ Characteristics } & \multirow[t]{2}{*}{ All $(n=60)$} & \multicolumn{2}{|c|}{ Preexisting rheumatic disorders } & \multicolumn{2}{|l|}{ Gender } \\
\hline & & Absent $(n=52)$ & Present $(n=8)$ & Male $(n=26)$ & Female $(n=34)$ \\
\hline \multicolumn{6}{|l|}{ Age (in years) } \\
\hline Up to 40 & $27(45.0)$ & $23(44.2)$ & $4(50.0)$ & $14(53.8)$ & $13(38.2)$ \\
\hline$>40$ & $33(55.0)$ & $29(55.8)$ & $4(50.0)$ & $12(46.2)$ & $21(61.8)$ \\
\hline Women & $34(56.7)$ & $27(51.9)$ & $7(87.5)$ & & \\
\hline Comorbidity & $30(50.0)$ & $24(46.1)$ & $6(75.0)$ & $9(34.6)$ & $21(61.8)$ \\
\hline No preexisting rheumatic disorders & $52(86.7)$ & & & $25(96.1)$ & $27(79.4)$ \\
\hline lgG positive & $19(31.7)$ & $16(30.8)$ & $3(37.5)$ & $6(23.1)$ & $13(38.2)$ \\
\hline \multicolumn{6}{|l|}{ Type of joint involvement } \\
\hline Only pain & $39(65.0)$ & $34(65.4)$ & $5(62.5)$ & $18(69.2)$ & $21(61.8)$ \\
\hline Pain with swelling & $21(35.0)$ & $18(34.6)$ & $3(37.5)$ & $8(30.8)$ & $13(38.2)$ \\
\hline \multicolumn{6}{|l|}{ Laboratory finding } \\
\hline Anti CCP & $1(1.7)$ & $0(0.0)$ & $1(1.9)$ & $1(3.8)$ & $0(0.0)$ \\
\hline HLA B27 & $2(3.4)$ & $1(1.9)$ & $1(1.9)$ & $1(3.8)$ & $1(2.9)$ \\
\hline Anti CCP + HLA B27 & $1(1.7)$ & $1(1.9)$ & $0(0.0)$ & $1(3.8)$ & $0(0.0)$ \\
\hline $\mathrm{RF}$ & $2(3.3)$ & $1(1.9)$ & $1(1.9)$ & $1(3.8)$ & $1(2.9)$ \\
\hline \multicolumn{6}{|l|}{ Ultra-sonographic findings } \\
\hline Tenosynovitis & $19(31.7)$ & $19(36.5)$ & $0(0.0)$ & $9(34.6)$ & $10(29.4)$ \\
\hline Synovial hypertrophy & $8(13.3)$ & $8(15.4)$ & $0(0.0)$ & $2(7.7)$ & $6(17.6)$ \\
\hline Tendinitis & $7(11.7)$ & $6(11.5)$ & $1(1.9)$ & $5(19.2)$ & $2(5.9)$ \\
\hline Median nerve entrapment & $4(6.7)$ & $4(7.7)$ & $0(0.0)$ & $1(3.8)$ & $3(8.8)$ \\
\hline Joint effusion & $3(5.0)$ & $2(3.8)$ & $1(1.9)$ & $1(3.8)$ & $2(5.9)$ \\
\hline Bursitis & $2(3.3)$ & $2(3.8)$ & $0(0.0)$ & $1(3.8)$ & $1(2.9)$ \\
\hline \multicolumn{6}{|l|}{ X-ray finding } \\
\hline Unilateral sacroiliitis & $1(1.7)$ & $0(0.0)$ & $1(1.9)$ & $0(0.0)$ & $1(2.9)$ \\
\hline Bilateral sacroiliitis & $4(6.7)$ & $4(7.7)$ & $0(0.0)$ & $1(3.8)$ & $3(8.8)$ \\
\hline Functional disability at one year & $(n=21)$ & $(n=17)$ & $(n=4)$ & $(n=9)$ & $(n=12)$ \\
\hline Mild & $18(85.7)$ & $15(28.8)$ & $3(37.5)$ & $8(30.8)$ & $10(29.4)$ \\
\hline Moderate & $2(3.3)$ & $2(3.8)$ & $0(0.0)$ & $1(3.8)$ & $1(2.9)$ \\
\hline Severe & $1(1.7)$ & $0(0.0)$ & $1(1.9)$ & $0(0.0)$ & $1(2.9)$ \\
\hline
\end{tabular}


Table 5 Types of arthritis and pain characteristics of the patients with Chronic Chikungunya arthritis $(n=60)$

\begin{tabular}{llcll}
\hline Type of arthritis & $\begin{array}{l}\text { Oligoarthralgia } \\
\boldsymbol{n}(\%)\end{array}$ & $\begin{array}{l}\text { Polyarthralgia } \\
\boldsymbol{n}(\%)\end{array}$ & $\begin{array}{l}\text { Mono/Oligoarthritis } \\
\boldsymbol{n}(\%)\end{array}$ & $\begin{array}{l}\text { Polyarthritis } \\
\boldsymbol{n}(\%)\end{array}$ \\
\hline Undifferentiated Arthritis $(n=35)$ & $2(5.7)$ & $21(60.0)$ & $4(11.4)$ & $8(22.8)$ \\
Spondyloarthritis $(n=10)$ & $0(0.0)$ & $6(60.0)$ & $1(10.0)$ & $3(30.0)$ \\
Rheumatoid Arthritis $(n=7)$ & $0(0.0)$ & $2(28.6)$ & $0(0.0)$ & $5(71.4)$ \\
Preexisting Arthritis* $(n=8)$ & $0(0.0)$ & $4(50.0)$ & $0(0.0)$ & $4(50.0)$ \\
\hline
\end{tabular}

*Preexisting Arthritis = Pre-existing spondyloarthritis, Pre-existing rheumatoid arthritis, Pre-existing osteo-arthritis arthritis

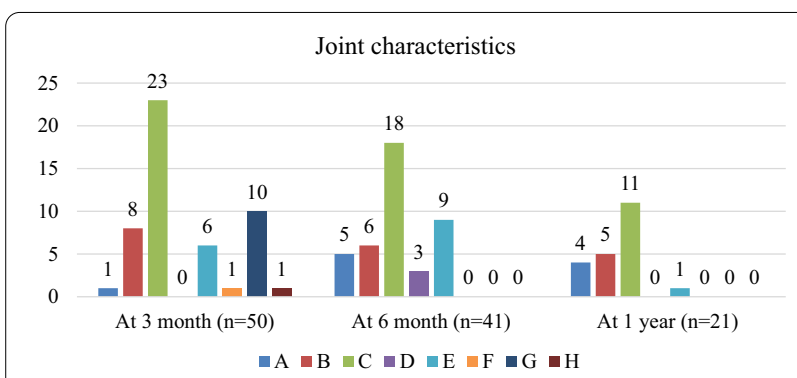

Fig. 3 Trend of arthralgia and arthritis among patients with Chronic CHIK arthritis for one year follow up. A Monoarthralgia, $B$ Oligoarthralgia, C Polyarthralgia, D Oligoarthritis, E Polyarthritis, $F$ Oligoarthralgia \& monoarthritis, G Polyarthralgia \& oligoarthritis, H Polyarthralgia \& Polyarthritis

Table 6 Predictors of post-Chikungunya- chronic inflammatory rheumatism ( $\mathrm{PCHIK-CIR)}$

\begin{tabular}{|c|c|c|c|c|}
\hline Variables & $\begin{array}{l}\text { Acute } \\
n(\%)\end{array}$ & $\begin{array}{l}\text { Chronic } \\
n(\%)\end{array}$ & $\begin{array}{l}\text { Crude OR } \\
(95 \% \mathrm{CI})\end{array}$ & $\begin{array}{l}\text { Adjusted OR } \\
(95 \% \mathrm{Cl})\end{array}$ \\
\hline \multicolumn{5}{|l|}{ Gender } \\
\hline Men & $47(64.4)$ & $26(35.6)$ & Ref. & \\
\hline Women & $36(51.4)$ & 34 (48.6) & $1.71(0.87-3.34)$ & $0.45(0.21-0.96)^{*}$ \\
\hline \multicolumn{5}{|l|}{ Comorbidity } \\
\hline Absent & $55(65.5)$ & $29(34.5)$ & Ref. & \\
\hline Present & $28(47.5)$ & $31(52.5)$ & $\begin{array}{l}2.10(1.06- \\
4.15)^{*}\end{array}$ & $0.62(0.29-1.31)$ \\
\hline \multicolumn{5}{|l|}{$\lg G$} \\
\hline Negative & $73(64.0)$ & $41(36.0)$ & Ref. & \\
\hline Positive & $10(34.5)$ & $19(65.5)$ & $\begin{array}{l}3.38(1.44- \\
7.96)^{*}\end{array}$ & $0.30(0.12-0.76)^{*}$ \\
\hline \multicolumn{5}{|c|}{ Functional disability } \\
\hline Mild & $37(46.8)$ & $42(53.2)$ & Ref. & \\
\hline $\begin{array}{l}\text { Moderate to } \\
\text { severe }\end{array}$ & $46(71.9)$ & $18(28.1)$ & $\begin{array}{l}0.34(0.17- \\
0.69)^{*}\end{array}$ & $3.45(1.62-7.40)^{*}$ \\
\hline
\end{tabular}

moderate to severe functional disability (adjusted OR: 3.45; CI: $1.62-7.40, p=0.001)$ were independent predictor of developing pCHIK-CIR (Table 6).

\section{Discussion}

Our study is the first prospective cohort study among Bangladeshi CHIKV patients to identify post-Chikungunya arthritis's clinical patterns and consequences. We evaluated patients in all three phases (acute, sub-acute, and chronic) of the disease, for which we were able to identify the predictors of chronicity. The study population consisted of $143 \mathrm{CHIKV}$-infected patients, where $41.9 \%$ of patients developed pCHIK-CIR within the oneyear follow-up, and polyarthralgia and mild functional disability were their main clinical presentation. In addition, female gender, positive IgG, and moderate to severe functional disability contributed to the development of chronicity.

More than half of the patients in the present study were male. Though female predominance was reported in several studies conducted in South India, Colombia, Venezuela, Sri Lanka [14, 32-36], male predominance was also reported in Singapore, Mayotte [37]. This inconsistency may relate to gender differences in exposure to infection due to community-specific habits, customs, or behaviors [38].

Most of the patients had a mild functional disability, while one-third had moderate and $10 \%$ had severe disability. This disability was significantly more in female patients. Female patients also had substantially more joint swelling and skin involvement than male patients, leading to more disability. This finding was comparable to the study of Kularatna et al. and Won et al. [36, 37]. Moreover, disabilities were more common in lower limbs. This led them to have difficulties doing common daily activities, maintaining hygiene, and walking. Rahim et al. [14] also stated that difficulties in doing these activities were due to the involvement of the lower limb (both soft-tissue and articular involvement).

Among these 143 patients of the present study, 41.9\% of patients developed chronic CHIK arthritis. The 3-year longitudinal study conducted in La Reunion reported that $60 \%$ of patients experienced symptoms of arthralgia [39]. Long-term arthralgias were typically polyarthralgia (70\%), usually symmetrical (90\%). Chronic inflammatory arthritis was uncommon in the rural community of India ( $0.3 \%$ at one year) [15]. In Colombia, at least half of the 
patients developed chronic rheumatologic sequelae [40]. Chronic arthritic disability was documented $45 \%$, where polyarthritis and arthralgia were common features in the village of Sri Lanka [36]. A systematic review and metaanalysis showed that the pooled prevalence of pCHIKCIR at 18 selected studies among 5,702 patients was $40.2 \%$. From studies derived from India, the prevalence was $27.3 \%$, while the prevalence was $50.2 \%$ from France. The systematic review and meta-analysis of RodriguezMorales et al. reported that the prevalence of pCHIK chronic arthritis was $13.7 \%$ [12]. These discrepancies might result from different strains of CHIKV in other world regions. Alternatively, reported differences might result from the different genetic backgrounds of these populations. As joint pain is considered a subjective symptom, it might also reflect a difference in patients' pain threshold or reporting from physicians, thus reflecting differences in health care practices.

The majority had undifferentiated arthritis among these chronic patients, while 7 (11.7\%) developed rheumatoid arthritis. Among the RA patients, two patients were Rheumatoid Factor positive (14.3\%). Several authors reported variable reports regarding Rheumatoid factors in individuals who had CHIKV infection [15, 41, 42]. Manimunda et al. tested the Rheumatoid Factor in chronic patients where they found no patient with positive Rheumatoid Factor [43]. Anti-CCP was positive in two patients. However, the case report of Lynch and Pegler [44] reported that an individual with chronic CHIKV infection was anti-CCP negative. Though there is variability in Rheumatoid Factor and anti-CCP in different countries, this should not be ignored as these factors worsen the disabilities.

Tenosynovitis was present in $30.0 \%$ of patients of the present study. Other studies also found tenosynovitis as an early symptom [44-47]. Besides this, synovial hypertrophy and tendinitis were also found in some patients. Two patients developed bursitis, which is documented in literature as an early symptom $[45,46]$. Three female and one male patient (12.5\%) had median nerve entrapment, suggestive of carpal tunnel syndrome (CTS). Studies conducted in Sri Lanka and Thailand also found female preponderance regarding Carpal tunnel syndrome $[36,44]$.

The time of joint affection in CHIK infection is uncertain. Javelle reported that $\mathrm{pCHIK-CIR}$ could persist even after 6 years since acute infection in 59\% of patients [11]. After one year, 21 patients of the present study had functional disabilities, where 11 had persistent pain for this long duration. In rural South India, 36.3\% of patients were found to have persistent pain after 18 months [14]. One female and one male patient of the present study had a moderate functional disability which developed undifferentiated arthritis and spondyloarthritis. Severe functional disability was present in one female who had pre-existing spondyloarthritis. A history of the previous rheumatic musculoskeletal disorder increased the probability of severe disability by more than two times in patients with persistent pain. Post-CHIKV infection exerts more negative effects on the previous disease, contributing to severe disability [14]. As different studies found chronicity at different times, long-term follow-up should follow.

In the present study, patients with undifferentiated arthritis had mainly polyarthralgia. One-fourth had polyarthritis, while oligoarthralgia, monoarthritis, and oligoarthritis were also present in some patients. Rahim et al. found that patients with undifferentiated arthritis had monoarthritis [14]. Patients with spondyloarthritis, pre-existing arthritis, and rheumatoid arthritis had several joint involvements such as polyarthralgia or polyarthritis. After three months, less than half of the patients had polyarthralgia, and one-fifth had polyarthralgia \& oligoarthritis. After six months, half of the patients had polyarthralgia, while at one year, polyarthralgia was present in 11 (52.4\%) patients. Patients should be informed that return to regular activity would progressively occur in many cases. They could be benefitted from sharing information about the possible chronic symptoms and the unpredictable course of the disease.

Laise de Moraes et al. described a scoring system, SHERA (Sex, Hypertension, Edema, Retroocular pain, Age), to screen acutely CHIKV-infected patients at elevated risk of chronic arthralgia [48]. We found female gender, positive IgG, and moderate to severe functional disability as the contributory factor in chronicity development. None of our patients had retroocular pain.

The systematic review of van Aalst et al. female gender was a risk factor for chronification [49]. The present study also found a significant association between pCHIK-CIR and the female gender. Since most women spend most of their time in and around the home, interrupting vector habitats near houses might be a helpful way to control epidemics. A significant association was found between comorbidity and development of pCHIK-CIR, which was consistent with Schilte et al. [39]. Badawi et al. also reported that comorbidities like hypertension and diabetes might contribute to the severe outcome of CHIKV [50]. Patients who were IgG positive and had moderate to severe functional disability in the acute stage significantly developed pCHIK-CIR. Gerardin et al. identified severe rheumatic involvement (fever, at least six joints plus four other symptoms) at presentation and CHIKVspecific IgG titers as the main risk factors for relapsing or lingering rheumatic manifestations [51]. The association between chronicity and severity of the initial presentation was also reported in Mexican and Indian patients 
$[52,53]$. All the patients with pre-existing RA had developed pCHIK-CIR. Moro et al. described the long-term clinical course and outcome of CHIKV following a Chikungunya (CHIKV) outbreak in Italy. They reported that persons with a history of rheumatologic diseases had higher anti-CHIKV IgG titers. At one year, the history of rheumatologic conditions was significantly associated with joint pain [54].

\section{Strength and limitations of the study}

All the study subjects were followed up for one year. The rheumatologist confirmed the diagnosis. Only the confirmed cases of CHIKV infection were included. The sample size was relatively small. Therefore, musculoskeletal ultrasonography was not done in all patients.

\section{Conclusions}

In conclusion, more than one-third of the CHIKV infected patients remained symptomatic after one year of the CHIKV outbreak in 2017. Polyarthralgia was the predominant clinical feature. Mild functional disability was also observed in a significant number of patients. Female patients with comorbidity, positive Ig G, moderate to severe functional disability, and pre-existing RA had significantly more chance of having pCHIK-CIR. Therefore, an integrated care plan should be made for patients with acute CHIK consisting of follow-up appointments with clinicians to identify pCHIK rheumatic disorders quickly and manage them accordingly. Above all, mass public awareness should be created to control the Aedes mosquito to reduce the disease burden.

\begin{abstract}
Abbreviations
ASAS: Assessment of Spondyloarthritis International Society; CHIKV: Chikungunya virus; CHIKF: Chikungunya fever; Cl: Confidence interval; CTS: Carpal tunnel syndrome; DMARDs: Disease-modifying antirheumatic drugs; HAQ: Health assessment questionnaire; OR: Odds ratios; pCHIK-CIR: Post-chikungunyachronic inflammatory rheumatism; RA: Rheumatoid arthritis; SpA: Spondyloarthritis; STROBE: Strengthening the Reporting of Observational Studies in Epidemiology; UA: Undifferentiated arthritis; WHO: World Health Organization; PDUS: Power doppler ultrasonography.
\end{abstract}

\section{Acknowledgements}

We thank all the patients for their willingness to participate in the study and express our gratitude to Prof. Syed Atiqul Haq, Prof. Md. Nazrul Islam and all the faculty members of the Rheumatology Department, BSMMU, for their cooperation. We also express our profound gratitude to Colonel Md. Saydur Rahman, ADMS 19th Infantry Division, Ghatail Cantonment, and Mr. Md. Helal Morshed, Statistician (CGO-1), Armed Forces Medical Institute, Dhaka Cantonment for cordial support.

\section{Authors' contributions}

MRC, MMH and SH conceived and designed the research protocol; MRC, MAI, $\mathrm{MMH}$ and SH prepared the questionnaire; SY and SH collected the data; MRC, $\mathrm{MAl}, \mathrm{MMH}$ and $\mathrm{SH}$ performed the patient evaluations; MRC, MAl, and $\mathrm{MMH}$ conducted the ultra-sonograms; $\mathrm{SH}$ and FH performed data entry, data analysis and interpreted the results; $\mathrm{SH}$ and FH drafted the manuscript, and MRC and MMZ critically reviewed, edited, and revised the manuscript. All authors read and approved the final manuscript.
Funding

The authors have no support or funding to report.

\section{Availability of data and materials}

The datasets used and analyzed during the current study are available from the corresponding author on reasonable request.

\section{Declarations}

\section{Ethics approval and consent to participate}

Study approval was obtained from the Institutional Review Board of BSMMU (BSMMU/2017/11262). Informed written consent was taken from the adult patients. A complete assurance was given that all information would be kept confidential. The right was being given to the patients not to participate and to discontinue participation at any time in the study with consideration/ without penalty. The Declaration of Helsinki's ethical guidelines were followed in the study.

\section{Consent for publication}

Not applicable.

\section{Competing interests}

The authors declare that they have no competing interests.

\section{Author details}

${ }^{1}$ Department of Rheumatology, Bangabandhu Sheikh Mujib Medical University (BSMMU), Shahbag Avenue, Dhaka, Bangladesh. ${ }^{2}$ Freelance Researcher, Dhaka, Bangladesh. ${ }^{3}$ WHO Bangladesh, Dhaka, Bangladesh.

Received: 14 November 2021 Accepted: 23 February 2022

Published online: 08 March 2022

\section{References}

1. Robinson MC. There was an epidemic of virus disease in Southern Province, Tanganyika territory, in 1952-1953. Trans R Soc Trop Med Hyg. 1955:49:28-32.

2. McSweegan E, et al. The global virus network: challenging chikungunya. Antivir Res. 2015;120:147-52.

3. Icddr,b. First identified an outbreak of chikungunya in Bangladesh, 2008. Health Sci Bull. 2009;7:1-6.

4. Khatun S, et al. An outbreak of chikungunya in rural Bangladesh, 2011. PloS Negl Trop Dis. 2015;9:e0003907.

5. Salje $\mathrm{H}$, et al. How social structures, space, and behaviors shape the spread of infectious diseases using chikungunya as a case study. Proc Natl Acad Sci. 2016;113:13420-5.

6. UN-Habitat (2017) "UN-Habitat Data Collections." http://urbandata.unhab itat.org/download-data/\#/indicators/9. Accessed Feb 2019

7. Jain M, Rai S, Chakravarti A. Chikungunya: a review. Trop Doct. 2008; $38: 70-2$

8. Powers AM. Chikungunya. Clin Lab Med. 2010;30:201-19.

9. Rolph MS, Foo SS, Mahalingam S. Emergent chikungunya virus and arthritis in the Americas. Lancet Infect Dis. 2015;15:1007-8.

10. Chow A, et al. Persistent arthralgia induced by Chikungunya virus infection is associated with interleukin- 6 and granulocyte-macrophage colony-stimulating factor. J Infect Dis. 2011;203:149-57.

11. Javelle $E$, et al. Specific management of post-chikungunya rheumatic disorders: a retrospective study of 159 cases in Reunion Island from 2006-2012. PLoS Negl Trop Dis. 2015;9:e0003603.

12. Rodríguez-Morales AJ, et al. Prevalence of post-chikungunya infection chronic inflammatory arthritis: a systematic review and meta-analysis. Arthrit Care Res. 2016;68:1849-58.

13. McCarthy MK, Morrison TE. Chronic chikungunya virus musculoskeletal disease: what are the underlying mechanisms? Future Microbiol. 2016;11:331-4.

14. Rahim AA, Thekkekara RJ, Bina T, et al. Disability with persistent pain following an epidemic of chikungunya in rural South India. J Rheumatol. 2016:43:440-4. 
15. Chopra A, et al. Acute Chikungunya and persistent musculoskeletal pain following the 2006 Indian epidemic: a 2-year prospective rural community study. Epidemiol Infect. 2012;140:842-50.

16. Mogami R, et al. Ultrasonography of hands and wrists in the diagnosis of complications of chikungunya fever. J Med Ultrasound. 2018;37:511-20.

17. Chowdhury F, et al. Chikungunya fever: an emerging threat to Bangladesh. J Med. 2012;13:60-4

18. Hassan R, et al. Chikungunya-an emerging infection in Bangladesh: a case series. J Med Case Reports. 2014:8:67.

19. Hossain MS, et al. Chikungunya outbreak (2017) in Bangladesh: Clinical profile, economic impact and quality of life during the acute phase of the disease. PloS Negl Trop Dis. 2018;12:e0006561.

20. Von Elm E, et al. The Strengthening the Reporting of Observational Studies in Epidemiology (STROBE) statement: guidelines for reporting observational studies. J Clin Epidemiol. 2008;61:344-9.

21. Chikungunya, WHO. Fact sheet. 2017. https://www.who.int/news-room/ fact-sheets/detail/chikungunya. Accessed Feb 2018.

22. Brito CA, et al. Pharmacologic management of pain in patients with Chikungunya guidelines. Rev Soc Bras Med Trop. 2016;49:668-79.

23. Simon F, et al. French guidelines for managing chikungunya (acute and persistent presentations). Med Mal Infect. 2015;45:243-63.

24. Aletaha D, et al. 2010 rheumatoid arthritis classification criteria: an American College of Rheumatology/European League Against Rheumatism collaborative initiative. Ann Rheum Dis. 2010;62:2569-81.

25. Rudwaleit MV, et al. The Assessment of SpondyloArthritis International Society classification criteria for peripheral spondyloarthritis and for spondyloarthritis in general. Ann Rheum Dis. 2011;70:25-31.

26. van der Linden S, Valkenburg HA, Cats A. Evaluation of diagnostic criteria for ankylosing spondylitis (A Proposal for Modification of the New York Criteria). Arthritis Rheumatol. 1984;27:361-8.

27. Smolen JS. Undifferentiated early inflammatory arthritis in adults. UpToDate 2021. https://www.uptodate.com/contents/undifferentiated-earlyinflammatory-arthritis-in-adults/print. Accessed Feb 2021.

28. Costantino $F$, et al. EULAR recommendations for the reporting of ultrasound studies in rheumatic and musculoskeletal diseases (RMDs). Ann Rheum Dis. 2021;80:840-7.

29. Naredo $E$, et al. Power Doppler ultrasonographic monitoring of response to anti-tumor necrosis factor therapy in patients with rheumatoid arthritis. Arthritis Rheum. 2008;58:2248-56.

30. Islam N, et al. Cross-cultural adaptation, and validation of a Bengali Health Assessment Questionnaire for use in rheumatoid arthritis patients. Int J Rheum Dis. 2013:16:413-7.

31. Bruce B, Fries JF. The Stanford Health Assessment Questionnaire: a review of its history, issues, progress, and documentation. J Rheumatol. 2003;30:167-78.

32. Rodríguez-Morales AJ, et al. Prevalence of post-chikungunya infection chronic inflammatory arthritis: a systematic review and meta-analysis. Arthritis Care Res. 2016;68:1849-58.

33. Rueda JC, et al. Demographic and clinical characteristics of chikungunya patients from six Colombian cities, 2014-2015. Emerg Microbes Infect. 2019:8:1490-500.

34. Mathew AJ, et al. Rheumatic-musculoskeletal pain and disorders in a naïve group of individuals 15 months following a Chikungunya viral epidemic in south India: a population-based observational study. Int J Clin Pract. 2011;65:1306-12.

35. Huits R, et al. Chikungunya virus infection in Aruba: Diagnosis, clinical features and predictors of post-chikungunya chronic polyarthralgia. PLoS ONE. 2018;13:e0196630

36. Kularatne SA, et al. Epidemiology, clinical manifestations, and long-term outcomes of a major outbreak of chikungunya in a hamlet in Sri Lanka, in 2007: a longitudinal cohort study. J Trop Med. 2012. https://doi.org/10. 1155/2012/639178

37. Win MK, et al. Chikungunya fever in Singapore: acute clinical and laboratory features, and factors associated with persistent arthralgia. J Clin Virol. 2010;49:111-4.

38. Sissoko D, et al. Seroprevalence and risk factors of chikungunya virus infection in Mayotte, Indian Ocean, 2005-2006: a population-based survey. PLoS ONE. 2008;3:e3066.

39. Schilte $C$, et al. Chikungunya virus-associated long-term arthralgia: a 36-month prospective longitudinal study. PLoS Negl Trop Dis. 2013;7:e2137.
40. Rodriguez-Morales AJ, et al. Post-chikungunya chronic inflammatory rheumatism: Results from a retrospective follow-up study of 283 adult and child cases in La Virginia, Risaralda, Colombia. F1000Res. 2016;5:360.

41. Bouquillard É, Combe B. A report of 21 cases of rheumatoid arthritis following Chikungunya fever. A mean follow-up of two years. Joint Bone Spine. 2009;76:654-7.

42. Morrison JG. Chikungunya fever. Int J Dermatol. 1979;18:628-9.

43. Manimunda SP, et al. Clinical progression of chikungunya fever during acute and chronic arthritic stages and the changes in joint morphology as revealed by imaging. Trans R Soc Trop Med Hyg. 2010;104:392-9.

44. Lynch N, Pegler RE. Persistent arthritis following Chikungunya virus infection. NZ Med J. 2010;123:79-81.

45. Simon F, et al. Chikungunya infection: an emerging rheumatism among travelers returned from Indian Ocean Islands Report of 47 cases. Medicine. 2007;86:123-37.

46. Parola P, Simon F, Oliver M. Tenosynovitis and vascular disorder associated with Chikungunya virus-related rheumatism. Clin Infect Dis. 2007:45:801-2

47. Malvy D, et al. Destructive arthritis in a patient with chikungunya virus infection with persistent specific IgM antibodies. BMC infect dis. 2009;9:200

48. de Moraes L, Cerqueira-Silva T, Nobrega V, et al. A clinical scoring system to predict long-term arthralgia in Chikungunya disease: a cohort study. PLoS Negl Trop Dis. 2020;14: e0008467. https://doi.org/10.1371/journal. pntd.0008467.

49. van Aalst M, Nelen CM, Goorhuis A, et al. Long-term sequelae of chikungunya virus disease: a systematic review. Travel Med Infect Dis. 2017;15:8-22. https://doi.org/10.1016/j.tmaid.2017.01.004.

50. Badawi A, et al. Prevalence of chronic comorbidities in chikungunya: a systematic review and meta-analysis. Int J Infect Dis. 2018;67:107-13.

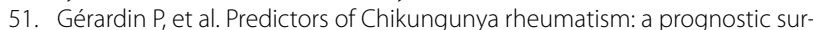
vey ancillary to the TELECHIK cohort study. Arthritis Res Ther. 2013;15:R9.

52. Murillo-Zamora E, et al. Persistent arthralgia and related risks factors in laboratory-confirmed cases of Chikungunya virus infection in Mexico. Rev Panam Salud Publica. 2017:41:e72.

53. Ramachandran $V$, et al. Persistent arthralgia among Chikungunya patients and associated risk factors in Chennai. South India J Postgrad Med. 2014;60:3-6.

54. Moro ML, et al. Long-term chikungunya infection clinical manifestations after an outbreak in Italy: a prognostic cohort study. J Infect. 2012;65:165-72.

\section{Publisher's Note}

Springer Nature remains neutral with regard to jurisdictional claims in published maps and institutional affiliations.

Ready to submit your research? Choose BMC and benefit from

- fast, convenient online submission

- thorough peer review by experienced researchers in your field

- rapid publication on acceptance

- support for research data, including large and complex data types

- gold Open Access which fosters wider collaboration and increased citations

- maximum visibility for your research: over $100 \mathrm{M}$ website views per year

At BMC, research is always in progress.

Learn more biomedcentral.com/submissions 